\title{
Esthetic Management of Congenitally Missing Lateral Incisors With Single Tooth Implants: A Case Report
}

\author{
Abu-Hussein Muhamad*,Watted Nezar**,Abdulgani Azzaldeen*** \\ *University Of Naples Federic II, Naples, Italy, Department Of Pediatric Dentistry, University Of \\ Athens, Athens, Greece \\ **Department Of Orthodontics, Arab American University, Jenin, Palestine \\ ***Department Of Conservative Dentistry, Al-Quds University, Jerusalem, Palestine
}

\begin{abstract}
Congenitally missing teeth are frequently presented to the dentist. Interdisciplinary approach may be needed for the proper treatment plan. Several treatment options exist for the replacement of congenitally missing lateral incisors.This case report addresses the fundamental considerations related to replacement of a congenitally missing lateral incisor by a team approach.
\end{abstract}

Keywords: congenitally missing lateral incisor, interdisciplinary treatment, dental implant.

\section{Introduction}

The successful use of dental implants to replace missing teeth has been one of the most popular, exciting and evolving areas of clinical dentist. When implants are thought as a treatment option, treatment planning has become more complex for the dental practitioner, and an interdisciplinary team approach is recommended.[1,2] Interdisciplinary approach would involve a preprosthetic and orthodontic treatment and following consultations with an oral surgeon and a restorative dentist, implant treatment was selected as a treatment modality .[2,3,4,5]

The maxillary lateral incisor is the second most frequently missing tooth after the mandibular second premolar even though Muller et al. found that maxillary lateral incisors experience the most agenesis (not including third molars). Agenesis of the maxillary lateral incisor is also linked with anomalies and syndromes such as agenesis of other permanent teeth, microdontia of maxillary lateral incisors (peg laterals), palatally displaced canines and distal angulations of mandibular second premolars.[6,7] Absence of any tooth can cause treatment difficulties, but agenesis of the maxillary lateral incisor poses a unique set of restorative challenges. Because the maxillary lateral incisor is located in the esthetic zone, it is essential that bone height, papilla height, enamel color, and shape match the surrounding teeth. Clinicians attempt to maintain the proper anterior overbite, overjet and ideal interarch relationships of the canine teeth while creating enough space for a fixed partial denture or more commonly, an implant with a single crown restoration, but few treatment options are available for patients with agenesis of one or both maxillary lateral incisors. One option is to close the space(s) and restore the remaining teeth accordingly and the second is to open the space for a fixed partial denture or implant. $[7,8,9,10]$

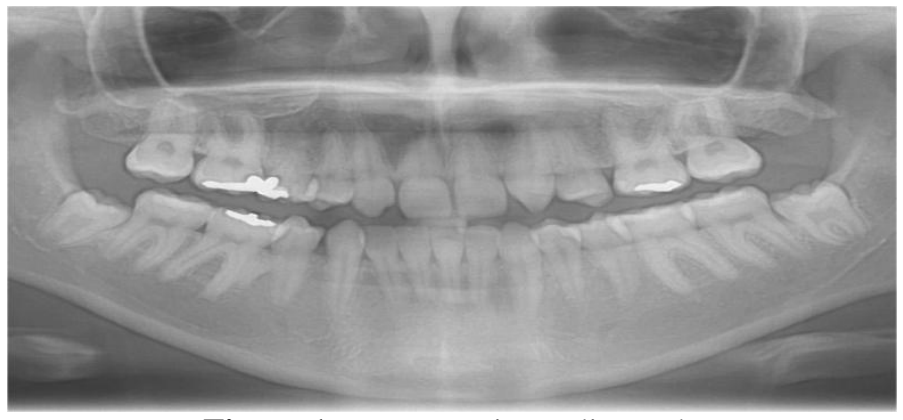

Figure 1: Pre-operative radiograph

Since the development of genetic mapping, Brook et al. have shown thatsome genes are implicated in the agenesis of teeth, including PAX9, MSX1 and AXIN2. The PAX9 gene is on chromosome 14 with a controlling factor for dental development and mutations related to missing teeth.[11] Brook et al. measured the tooth sizes on maxillary and mandibular dental casts in the test group, 10 people with a known PAX9 mutation in one family and 10 people in a control group matched for sex, age and ethnicity, who were not related to the test group and did not have the PAX9 mutation. Differences in the test group with the mutation and hypodontia were found; these teeth were significantly smaller than controls. Canines and first 
molars were least affected in the test group. [11]This contradicts Bailit's theory that genetics mostly affects the first tooth in each group: the central incisor, canine, first premolar and molar.[12] Brook et al. found that the second tooth in each group was more affected by the PAX9 mutation. The study concluded that the PAX9 mutation not only decreased tooth number, but also tooth size throughout the dentition.[11]

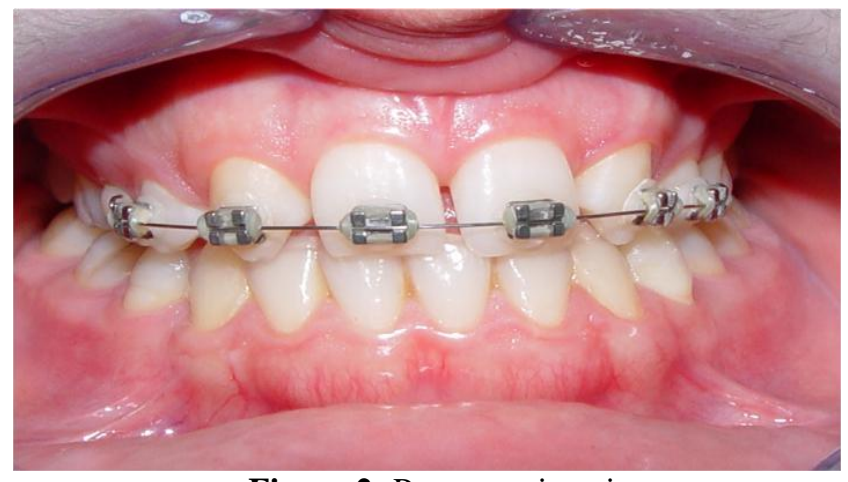

Figure 2: Pre-operative view

Peck, Peck and Kataja linked palatally displaced canines, transposition of mandibular lateral incisors and canines and maxillary canine and premolar transposition with agenesis. [13] They studied 161 subjects and found that patients with maxillary canine-first premolar transposition were 13 times more likely (26\%) to have agenesis of a maxillary lateral incisor. They agreed with Brook et al. that PAX9 and MSX1 mutations contribute to tooth agenesis20, however they also pointed out that the PAX9 and MSX1 genetic mutations are associated with posterior tooth agenesis while a strong causative gene mutation for anterior agenesis has yet to be found. Peck, Peck and Kataja believe signaling proteins such as bone morphogenic proteins (BMP) and fibroblast growth factor (FGF) may be responsible for agenesis early in embryonic development.[13] Arte et al. mapped the genes of 77 individuals in 3 generations of Finnish families to determine which genes affect incisorpremolar hypodontia.16 Thirty-one of the 77 participants expressed incisor-premolar hypodontia; however childrenunder the age of 6 were excluded from the study because hypodontia cannot reliably be ascertained at that age. They hypothesized that epidermal growth factor (EGF), epidermal growth factor receptor (EGFR), and fibroblast growth factor (FGF-3) genes were responsible for incisor-premolar hypodontia because: 1) EGF has been implicated in early tooth morphogenesis in mice, 2) EGFR is expressed in developing teeth from the bud stage onward, and 3) FGF-3 is intensely expressed in dental mesenchyme during cap and bell stages of tooth development. However, after extracting DNA from blood samples of the participants and running PCR procedures, results did not show that these genes are involved in incisor-premolar hypodontia.

They concluded that mutations in EGF, EGFR, FGF-3 and FGF-4 did not influence incisor-premolar hypodontia in the families studied.[14] Genetic factors appear to play a large role in tooth size and agenesis with the PAX9 and MSX1 mutations; however some authors suspect that the local environment is important factor.[7] EGF, EGFR, FGF-3 and FGF-4 are not shown to be linked to incisor-premolar agenesis, but it is possible that signaling factors early in embryologic development may contribute to agenesis. Through the work of Pirinen et al. and Arte et al., it is evident that incisorpremolar hypodontia is genetically inherited, with strong links to other dental anomalies such as palatally impacted canines. Incisor-premolar hypodontia is an autosomal dominant gene inheritance with incomplete penetrance. [14,15]

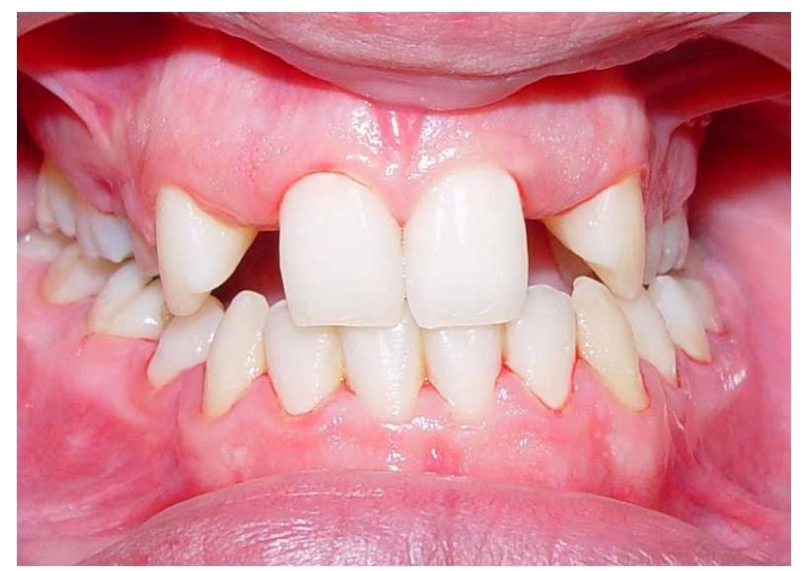

Figure 3: View of preoperative sites after the removal of orthodontic braces 
The incidence of congenitally missing maxillary lateral incisors has been reported to range from between $1 \%$ and $2 \% 1$ to as high as $5 \%$. Maxillary lateral incisors are the most common congenitally missing teeth after upper and lower second premolars. Sex differences have been found to be negligible, with slightly more females affected as compared with males. [16,17]Treatment options include orthodontic movement of cuspids into lateral incisor sites, prosthodontic restorations including fixed and removable prostheses and resinbonded retainers, and single tooth implants.[9] Implants do not require preparation of natural teeth and thus can be considered the most conservative approach. Orthodontic space opening may be necessary but on occasion can compromise esthetics, periodontal health, and function.[1,2]

Parents and professionals must often decide at a child's early age on how to cope with congenitally missing maxillary lateral incisors. Orthodontic treatment to create space for implants should not be initiated before the age of 13. This will avoid the potential for alveolar bone atrophy and the risk of relapse and subsequent retreatment.[3,4] The management of small restorative areas in the esthetic zone has posed significant problems for the implant and restorative team. The lack of bone available for the surgeon as well as the lack of restorative space available between the adjacent teeth makes tooth replacement with implants challenging for both the restorative dentist and the laboratory technician. In the past, patients with congenitally missing teeth or microdontia have been treated with resin-bonded bridges, removable retainers, or cantilever crowns to avoid the use of standard-diameter implants and prosthetics in this area.[1,3] The two common treatment options include orthodontic space opening for future restorations or orthodontic space closure utilizing the adjacent permanent canine teeth. With a paradigm shift in the patient expectations towards functional as well as esthetically appealing replacements for edentulism, the implant based oral rehabilitation has emerged as a sole winner in fulfilling all aspects of patient needs.[1,2,3,4,5]

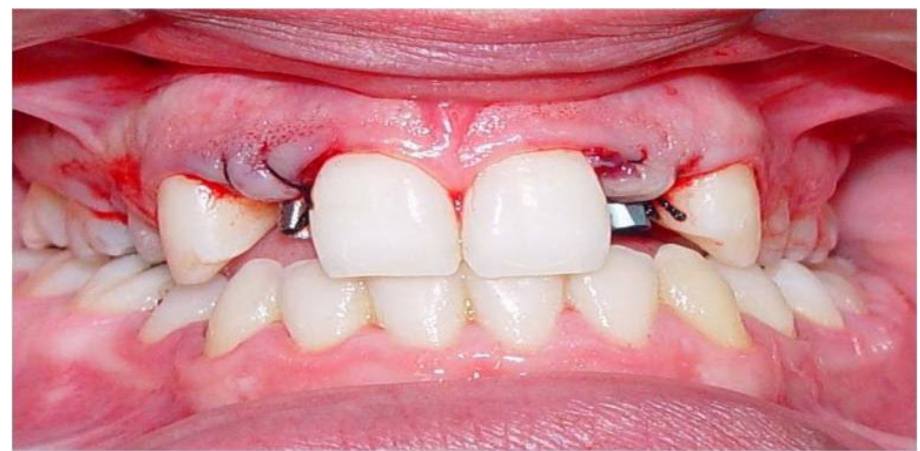

Figure 4: Facial view immediately after the insertion of two Abutment

The orthodontist plays a key role in determining and establishing space requirements for patients with congenitally missing maxillary lateral incisors .However, the implant based treatment option in such patients requires an interactive and interdisciplinary management approach. $[1,3,5]$ This interdisciplinary approach may involve preprosthetic orthodontic treatment following consultations with an oral surgeon or periodontist and restorative dentist to ensure orthodontic alignment will facilitate the surgical, implant and restorative treatment. Too often, surgeons attempting to place standard-diameter implants have forced the restorative team to manage these small dimensions with a lack of adequate prosthetics because of the size and diameter of the fixture head. Recently, manufacturers in the implant industry have offered a 3-mm diameter implant design to address these challenges. [3,4,5]Most of the implants available in the 3-mm size have been one-piece or unibody implants, which often necessitate conventional tooth-preparation techniques by the restorative team as well as standard cord-impression techniques for indexing the restorative margins. With some systems, there is no need for preparation due to a cervical marginal collar that can be captured utilizing a snap-in impression transfer.[16,17,18,19,20]

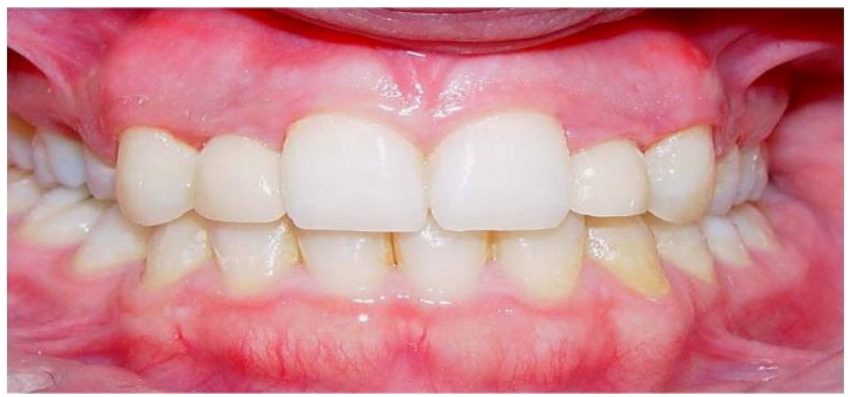

Figure 5: Zirconia crowns cemented 
Classically, congenitally missing lateral incisors can be restored in three ways.[5] A camouflage treatment modality can be performed by mesialization of the canine into the lateral incisor space and performing conservative reshaping of the canine to mimic the incisor.[1,2] A second treatment possibility is a space opening orthodontic approach, aiming to create adequate space for the placement of an osseointegrated implant in the incisal area or to allow the seating of a fixed partial denture.[5] The third option is orthodontic creation of space in the posterior area to allow the placement of an implant in the premolar area.

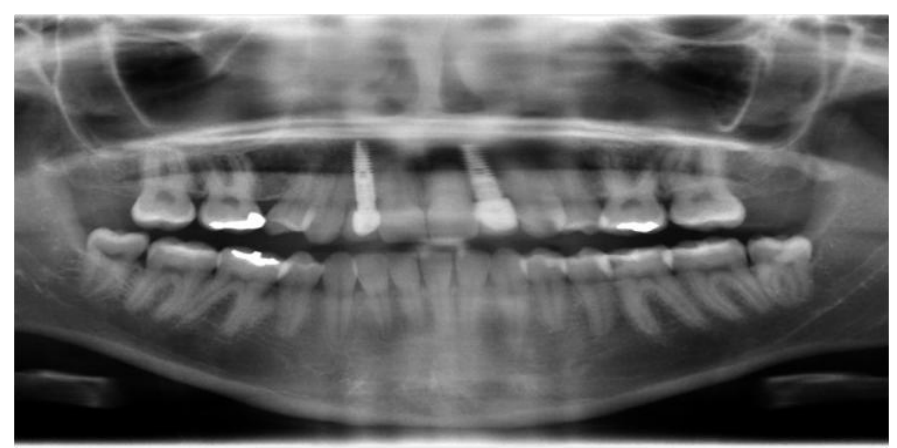

Figure 6: Post-operative radiograph

Implant therapy is an established treatment modality for the rehabilitation of single or multiple missing teeth with high implant success rates in the long term.8 Dental implants are able to provide a high esthetic outcome in very demanding clinical situations, such as the rehabilitation of missing teeth in the premaxilla.9 In the last few years, investigators have focused their efforts on determining a reliable method that is able to evaluate the esthetic outcome of an implant supported restoration objectively.

This case report addresses the fundamental considerations related to replacement of a congenitally missing lateral incisor by a team approach.

\title{
Case Report
}

The initial clinical exam revealed diastema, congenitally missing maxillary lateral incisors with the canines located in the lateral incisor positions, and the primary maxillary canines still located in their original positions.These aspects created not only esthetics deficiencies but also maloclussion. Therefore, a multidisciplinary treatment was suggested to restore both esthetics and function.[9]

This case was a 20-year-old female who had congenitally missed maxillary left lateral incisor. Her chief complaint was diastema and missing her upper left and right laterals.

\author{
The treatment plan was: \\ - Initial therapy (SRP) \\ - Orthodontic therapy for alignment and achievement of sufficient space. \\ -Surgery: Ridge augmentation and implant placement. \\ - Prosthesis. \\ - SPT (Supportive periodontal therapy).
}

First by orthodontic therapy the space required for implant placement was achieved. Materials and Methods: Autogenous bone grafts are the best materials for limited ridge augmentations. They can be obtained in a number of ways and from several donor sites. In the present case report, the recipient sites were limited in size, and trephine drills (Trephine Bur Bone Harvest) were used to obtain the bone graft because the tomography showed that the ridge width was insufficient for implant placement. After anesthetizing the operation site with lidocaine, a crestal incision was performed. An envelope flap was retracted using blunt dissection to limit it to the graft site with no excessive extension. The periosteum was raised, intact and undamaged, from the bone. The flap was extended laterally to obtain enough space for the bone graft, and no vertical incision was made. We obtained bone from the internal oblique ridge area. A lingual sulcular incision was made to expose the donor site from the distal end of the first molar to the distal aspect of second (or third, if present) molar. A number 5 or 6 trephine drill was used as needed to harvest one or two pieces of bone.

Next, the donor site was sutured and compressed with damp gauze sponge. The bone blocks were placed inside the envelope flap at the recipient site. In some cases, the 


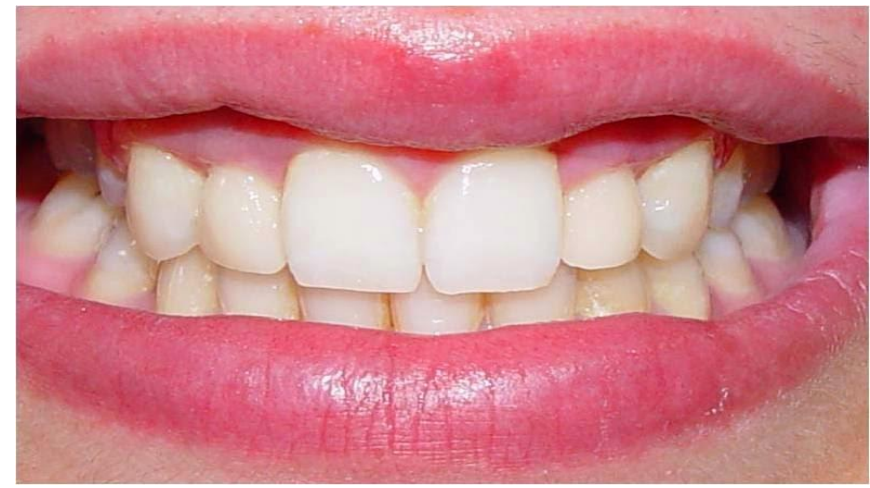

Figure 6: The implant-supported restoration after five years.

shape of the bone graft was adjusted to enhance its adaptation to the recipient site. Due to the limited extension of the flap and the orderly setting of the bone blocks, there was no need to use screws to secure the bone grafts in place.

The recipient site was then sutured and covered with periodontal dressing. From $24 \mathrm{~h}$ prior to the operation, patients were administered Amoxicillin $(500 \mathrm{mg}$ ) every $6 \mathrm{~h}$, and the treatment continued for 5-7 d. Chlorhexidine mouthwash $(0.2 \%$ solution) was prescribed for two weeks for all of the cases. Additionally, the patients were prescribed Acetaminophen plus Codeine. After a week, the sutures were removed. Six months later, the recipient site was reopened, and the bone width was measured, and one Implant with $3.8 \mathrm{~mm}$ diameter and $10 \mathrm{~mm}$ length was placed. Three months later, second stage surgery and aesthetic surgery for leveling of gingival margins was performed and final restoration was placed (Figs. 1- 6).

\section{Discussion}

For patients with congenitally missing lateral incisors, in addition to over-retained primary teeth, permanent canines may erupt or drift mesially into the edentulous space. If the space is to be opened orthodontically for ideal prosthesis, the canines will need to be moved distally, which may result in development of the alveolar ridge in the canine region $[2,3,5]$. In cases where the occlusion and esthetics of the canine in the lateral position are acceptable, closure of the lateral space by the mesially positioned canine may be the simplest alternative treatment option.However, in all the above congenital missing cases we considered space opening followed by fixed prosthesis would be more acceptable on aesthetic point of view [19,20,21].

Esthetics as well as occlusion must be considered in the final orthodontic positioning of the teeth adjacent to the edentulous space. To satisfy the "golden proportion" principle of esthetics, the space for the maxillary lateral incisor should be approximately two-thirds of the width of the central incisor.

However, if the patient is missing only one maxillary lateral incisor, the space required to achieve symmetrical esthetics and occlusion is primarily dictated by the width of the contralateral incisor [22].

The treatment outcome for this patient was very favorable, since it achieved a wider maxillary arch by opening space with placement of implant/prosthesis in the region of the maxillary canines and anatomic restoration of the maxillary right and left lateral incisors. This procedure provided better proportion of the tooth crowns in the maxillary anterior region as well as enhanced gingival contour, favoring an esthetic smile.[22-25] In this study we harvested bone grafts from external oblique ridge areas. Verdugoe et al. previously evaluated different areas of the mandible as bone graft donor sites and found that the second and third molar regions could provide good bone graft pieces with a mean thickness of $2.8 \pm 0.6 \mathrm{~mm}$.[26] We performed a simple, nonaggressive operation by designing an envelope flap limited to the edentulous area. The bone blocks that were harvested using trephine drills were placed into the envelope, and there was no need to secure the bone pieces.[1,3,5]

The advantages of this technique are its simplicity, the fact that there is no need for decortication of the recipient site, and no need for using screws. Previously, Acocella et al. used bone blocks obtained from the mandibular ramus for lateral augmentation and reported a $4 \pm 0.77 \mathrm{~mm}$ increase in ridge width . [27]Funaki et al. used distraction osteogenesis and bisection techniques to increase ridge width and reported a $2.7 \mathrm{~mm}$ increase for distraction and a $1.7 \mathrm{~mm}$ increase for bisection .[28] Antal used the same method for achievement of optimal emergence profile.[29]

Concerning the dental relationships between the maxillary and mandibular arches, there was a great improvement in terms of anterior tooth position and correction of the mandibular midline, highlighting the treatment benefits when considering the limitations of retreatment in an adult patient.[1-5,31,32] 
The overall treatment was long and dependent upon patient participation and comprehension. On the other hand, as a result of the use of this multidisciplinary approach, it was possible to create a good occlusal relationship that met with the patient's satisfaction.[30,31,32]

\section{Conclusions}

Orthodontic space closure and implant substitution of missing maxillary incisors produced similar satisfactory esthetic results. Neither of the treatments impaired temporomandibular joint function.

However, this case report demonstrate an acceptable increase in ridge width and this technique may be used successfully as an alternative to the current, invasive augmentation methods. Furthermore, successful restorative treatment involving implants depends on interdisciplinary treatment planning, preprosthetic orthodontic tooth alignment for achievement sufficient space, bone grafting for augmentation ridge width and implant surgery and prosthesis.

\section{References}

[1]. Abu-Hussein M., Abdulgani A., Watted N .Zahalka M.(2015); Congenitally Missing Lateral Incisor with Orthodontics, Bone Grafting and Single-Tooth Implant: A Case Report. Journal of Dental and Medical Sciences , 14(4),124-130 DOI: 10.9790/08531446124130

[2]. Abdulgani A.,. Kontoes N., Chlorokostas G.,Abu-Hussein M (2015).; ;Interdisciplinary Management Of Maxillary Lateral Incisors Agenesis With Mini Implant Prostheses: A Case Report; Journal of Dental and Medical Sciences ,14 (12) , 36-42 DOI: $10.9790 / 0853-141283642$

[3]. Abusalih A. , Ismail H, Abdulgani A., Chlorokostas G ., Abu-Hussein M .(2016); Interdisciplinary Management of Congenitally Agenesis Maxillary Lateral Incisors: Orthodontic/Prosthodontic Perspectives, Journal of Dental and Medical Sciences , 15 ( 1) , 90-99 DOI: 10.9790/0853-15189099

[4]. Abu-Hussein M., Watted N., Abdulgani A., BorbélyB.(2015); Modern Treatment for Congenitally Missing Teeth : A MultidisciplinaryApproach; INTERNATIONAL JOURNAL OF MAXILLOFACIAL RESEARCH, 1(2);179-190

[5]. Abu-Hussein M, Chlorokostas G, Watted N , Abdulgani A , Jabareen A, (2016),Pre-Prosthetic Orthodontic Implant for Management of Congenitally Unerupted Lateral Incisors - A Case Report Journal of Dental and Medical Sciences 2 .Vol 15 (2 ), 99-104 DOI: 10.9790/0853-152899104

[6]. Muller TP, Hill IN, Peterson AC, Blayney JR. (1970); A survey of congenitally missing permanent teeth. J Am Dent Assoc ;81:101-7.

[7]. Abu-Hussein M., Watted N., Yehia M., Proff P., Iraqi F.(2015); Clinical Genetic Basis of Tooth Agenesis, Journal of Dental and Medical Sciences ,14(12),68-77 DOI: 10.9790/0853-141236877

[8]. Abu-Hussein M., Watted N., Abdulgani A., Kontoes N(2015); Prosthodontic-Orthodontic Treatment Plan with TwoUnitCantilevered Resin-Bonded Fixed Partial Denture, IOSR-JDMS 2015,14(12), 131-136 DOI: 10.9790/0853-14124131136

[9]. Muhamad AH, Azzaldeen A, Nezar W, Mohammed Z. ( 2015); Esthetic Evaluation of Implants Placed after Orthodontic Treatment in Patients with Congenitally Missing Lateral Incisors. J Adv Med Dent Scie Res ;3(3):110-118.

[10]. Abdulgani M. , Abdulgani Az ., Abu-Hussein M .(2016); Two Treatment Approaches for Missing Maxillary Lateral Incisors: A Case Journal of Dental and Medical Sciences Volume 15, Issue 7 , 78-85 DOI: 10.9790/0853-150787885

[11]. Brook AH, et al. ( 2008); Tooth dimensions in hypodontia with a known PAX9

[12]. mutation. Archives of Oral Biol. ; doi:10.1016/j.archoralbio.2008.05.017

[13]. Bailit HL. ( 1975); Dental variation among populations: an anthropologic view. Dent Clin North Am ;19:125-139

[14]. Peck Se, Peck L, Kataja M. ( 2002); Concomitant occurrence of canine malposition and tooth agenesis: evidence of orofacial genetic fields. Am J Orthod Dentofacial Orthop ;122:657-60.

[15]. Arte S et al. ( 1996); Gene defect in hypodontia: exclusion of EGF, EGFR, and FGF-3 as candidate genes. J dent res 75(6):13461352

[16]. Pirinen S. Arte S, Apajalahti S. ( 1996); Palatal displacement of canine is genetic and related to congenital absence of teeth. J Dent Res ;75(10):1742-1746.

[17]. Abu-Hussein M., Watted N., Watted A., Abu-Hussein Y, Yehia M .Awadi O. , Abdulgani A . (2015); Prevalence of Tooth Agenesis in Orthodontic Patients at Arab Population in Israel, International Journal of Public Health Research ; 3(3): 77-82.

[18]. Muhamad Abu-Hussein, Nezar Watted, Abdulgani Azzaldeen, Mohammad Yehia, Obaida Awadi, Yosef Abu-Hussein. (2015); Prevalence of Missing Lateral Incisor Agenesis in an Orthodontic Arabs Population in Israel (Arab48). International Journal of Public Health Research. Vol. 3, No. 3, . 101-107.

[19]. Muhamad AH, Azzaldeen A (2012) Autotransplantation of Tooth in Children with Mixed Dentition. Dentistry 2:149. doi:10.4172/2161-1122.1000149

[20]. Abu-Hussein M. , Watted N . ,Abdulgani M ., Abdulgani Az .(2016); Tooth Autotransplantation; Clinical Concepts Journal of Dental and Medical Sciences, Vol 15 (7) 105-113 DOI: 10.9790/0853-15078105113

[21]. Abu-Hussein M ,1, Nezar W. , Azzaldeen A. , Abdulgani M(2016); Prevalence of Traumatic Dental Injury in Arab Israeli Community, Journal of Dental and Medical Sciences ;15 (7) , 91-98 DOI: 10.9790/0853-150719198

[22]. Kokich VO Jr, Kinzer GA. (2005)Managing congenitally missing lateral incisors

[23]. Part I: Canine substitution. J. Esthet Restor Dent ;17(1):5-10

[24]. L. Savarrio and G. T. McIntyre,(2005); "To open or to close space - that is the missing lateral incisor question," Dental Update, vol. no. 1, pp. 16-25.

[25]. Rosa M, Zachrisson BU.(2010; The space-closure alternative for missing maxillary lateral incisors: an update. J Clin Orthod. ;XLIV(9):540-549

[26]. Kokich VG.(2004); Maxillary lateral incisor implants: planning with the aid of

[27]. orthodontics. J Oral Maxillofac Surg. ;62:48-56.

[28]. Kokich VO Jr, Kinzer GA.(2005) Managing congenitally missing lateral incisors Part III: Single-tooth implants. J. Esthet Restor Dent ;17(4):202-10 
[29]. Verdugo F, Simonian K, Smith McDonald R, Nowzari H. Quantitation of mandibular ramus volume as a source of bone grafting. Clin Implant Dent Relat Res 2009; 11 Suppl 1:e32-7.

[30]. Acocella A, Bertolai R, Colafranceschi M, Sacco R.( 2010)Clinical, histological and histomorphometric evaluation of the healing of mandibular ramus bone block grafts for alveolar ridge augmentation before implant placement. J CraniomaxillofacSurg ; 38: 22230.

[31]. Funaki K, Takahashi T, Yamuchi K.( 2009)Horizontal alveolar ridge augmentation using distraction osteogenesis: comparison with a bone-splitting method in a dog model. Oral Surg Oral Med Oral Patho Oral Radiol Endod ;107:350-58.

[32]. Antal M. Replacing Congenitally Missing Central Incisor: A Case Report with a Special Method to Achieve the Optimal Emergence Profile. Surgery S11: 003. doi:10.4172/2161-1076.S11-003.

[33]. Abu-Hussein M, Watted N,( 2015); Congenitally Missing Upper Laterals. Clinical Considerations: Orthodontic Space Closure,Int Res Pub Med Sci, ;1(3):82-89

[34]. Abu-HusseinM.,Watted N. (2016); Maxillary Midline Diastema - Aetiology And Orthodontic Treatment- Clinical Review .Journal of Dental and Medical Sciences , 15(6),116-130 DOI: 10.9790/0853-150602116130

[35]. Abu-Hussein M ,Watted N ,Abdulgani A (2015);An Interdisciplinary Approach for Improved Esthetic Results in the Anterior Maxilla Diastema Journal of Dental and Medical Sciences ,14(12), 96-101,DOI: 10.9790/0853-1412896101 